\title{
What will move malaria control to elimination in South Africa?
}

\author{
D Moonasar, ${ }^{1} \mathrm{DrPh}$; N Morris, ${ }^{2} \mathrm{MSc}$; I Kleinschmidt, ${ }^{3} \mathrm{PhD}$; R Maharaj, ${ }^{2} \mathrm{PhD} ; \mathrm{J}$ Raman, ${ }^{2} \mathrm{PhD} ; \mathbf{N}$ T Mayet, ${ }^{4} \mathrm{MB}$ ChB; F G Benson, ${ }^{1} \mathrm{MB}$ ChB; \\ D N Durrheim, ${ }^{5,6} \mathrm{DrPh}$, FAFPHM; L Blumberg, ${ }^{7}$ MMed (Path Microbiol) \\ ${ }^{1}$ Malaria Directorate, National Department of Health, Pretoria, South Africa \\ ${ }^{2}$ Malaria Research Unit, South African Medical Research Council, Durban, South Africa \\ ${ }^{3}$ Department of Infectious Diseases and Epidemiology, London School of Hygiene and Tropical Medicine, London, United Kingdom \\ ${ }^{4}$ South African Regional Global Disease Detection Centre, National Institute for Communicable Disease, Johannesburg, South Africa \\ ${ }^{5}$ School of Public Health Medicine, University of Newcastle, Australia \\ ${ }^{6}$ Public Health and Tropical Medicine, James Cook University, Queensland, Australia \\ ${ }^{7}$ Division of Public Health Surveillance and Outbreak Response, National Institute for Communicable Diseases, Johannesburg, South Africa
}

Corresponding author: D Moonasar (moonad@health.gov.za)

In this supplement, several authors have shared lessons from the past and identified factors that led to the significant reductions in malaria morbidity and mortality during the past half-century in South Africa. In addition, strategies for achieving malaria elimination have been proposed. Here, we highlight the gaps that have been identified and make proposals for taking South Africa from malaria control to elimination.

S Afr Med J 2013;103(10 Suppl 2):801-806. DOI:10.7196/SAMJ.7445

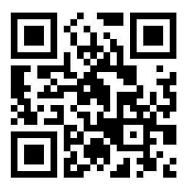

South Africa (SA) is one of 34 malaria-endemic countries currently targeting elimination of the disease - i.e. no local malaria transmission in a defined geographical area - with the goal to achieve elimination by $2018 .^{[1-3]}$ In 2009, the SA National Department of Health (NDoH) conducted a comprehensive malaria programme review, aimed at identifying gaps for eliminating the disease and developing a national elimination strategy for SA. ${ }^{[4]}$ During the programme review, it became evident that malaria transmission still occurred in 9 districts in SA: Capricorn, Mopani, Sekhukhune, Vhembe and Waterberg in Limpopo; Ehlanzeni District in Mpumalanga; and uMkhanyakude, uThungulu and Zululand districts in KwaZulu-Natal (Fig. 1).

\section{Malaria elimination continuum}

The World Health Organization (WHO) developed a malaria elimination continuum to assist control programmes in malariaendemic countries to determine their malaria status, using malaria incidence values. ${ }^{[2]}$ Countries with a malaria blood-slide positivity rate among fever cases $>5$ cases $/ 1000$ population at risk are considered to be in the malaria control stage; $<5$ and $>1$ case/ 1000 are in the preelimination stage; and at rates $<1$ case/ 1000 are categorised as being in the elimination stage (Fig. 2).

Based on routine malaria notification data, incidence of autochthonous malaria cases in the 9 endemic districts of SA for the financial year 2012/13 (April - March) was 0.18/1 000 population per annum $(n=919) \cdot{ }^{[5]}$ In the 3 endemic provinces, the incidence per 1000 population at risk for the same reporting period was 0.50 for Limpopo, 0.13 for Mpumalanga and 0.03 for KwaZulu-Natal. At present, malaria incidence in all malaria-endemic districts, with the exception of Vhembe District in Limpopo, meet the eliminationstage threshold of the WHO elimination continuum (Table 1). However, within districts are localities in which transmission is much higher.

\section{Gaps in the SA Malaria Control Programme}

Key gaps identified, and which must be addressed to achieve elimination, are:

- active surveillance in response to confirmed cases and treatment of identified cases, to interrupt local transmission

- sensitive diagnostic tests to detect low-level parasitaemias

- maintaining a high level of malaria awareness by communities and health workers, as the malaria prevalence decreases

- monitoring parasite drug and vector resistance, as these risks increases when malaria case numbers decrease

- supporting malaria control measures in neighbouring countries more effectively

- sustained funding in the face of reduced case numbers.

\section{Surveillance and case investigation}

Passive case notifications are posted weekly by telephone to the relevant district office. However, there is a lag-period of $1-2$ weeks before the data are entered electronically into a malaria information system (MIS). Only then is case investigation and follow-up initiated for all cases in an endemic district. Generally, this follow-up occurs within 2 days of data entry in the MIS. The total lag between case diagnosis and case investigation is therefore far in excess of the target of follow-up of within 48 hours under an elimination agenda, and the required turn-around is at present not achievable following existing protocols and procedures.

Pilot studies are underway in two subdistricts to evaluate the feasibility of implementing mobile phone short-message service (SMS)-based reporting to improve case notification turnaround times and enable investigation within a 48 -hour period. If successful, the approach may be adopted in districts classified as being within the elimination stage, and subsequently introduced in those in the pre-elimination stage.

The evolution of separate reporting systems (district health and MISs) at provincial level, regional differences in epidemiology and different 


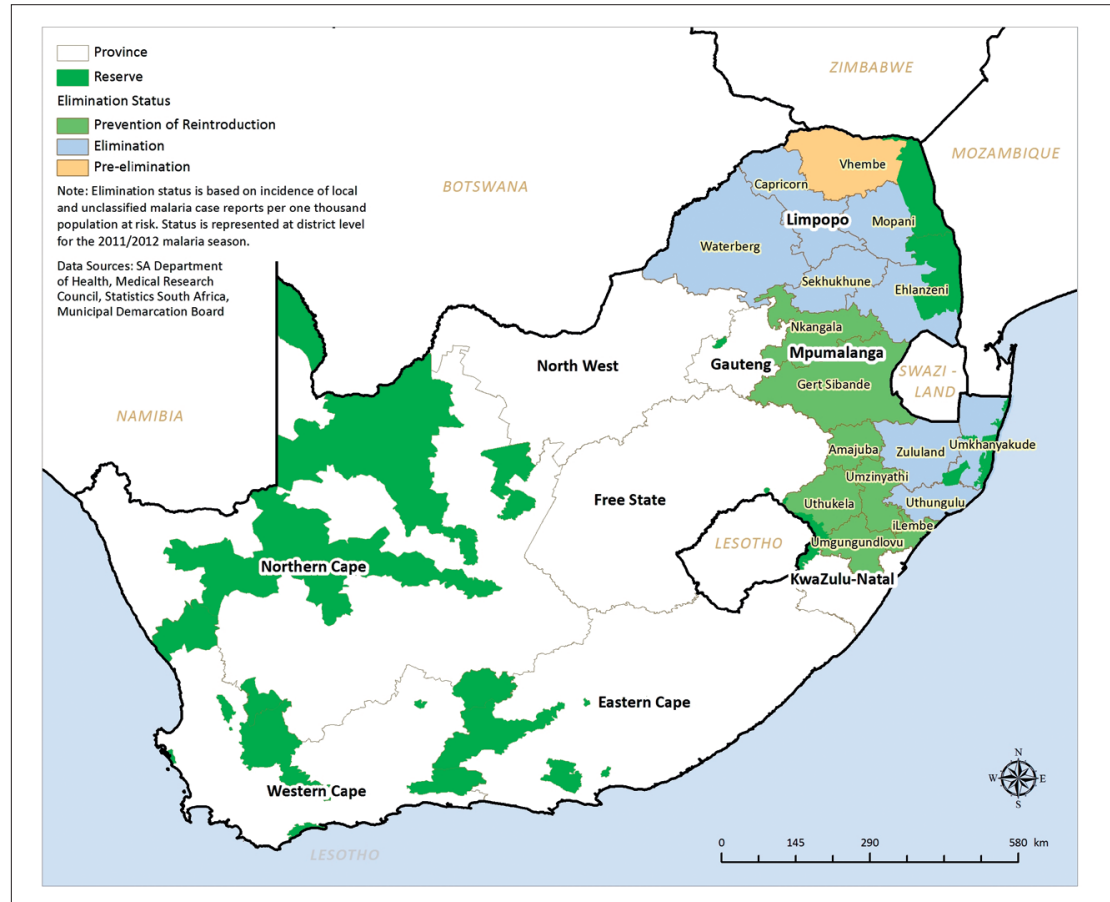

Fig. 1. SA malaria districts elimination status, 2011 - 2012.

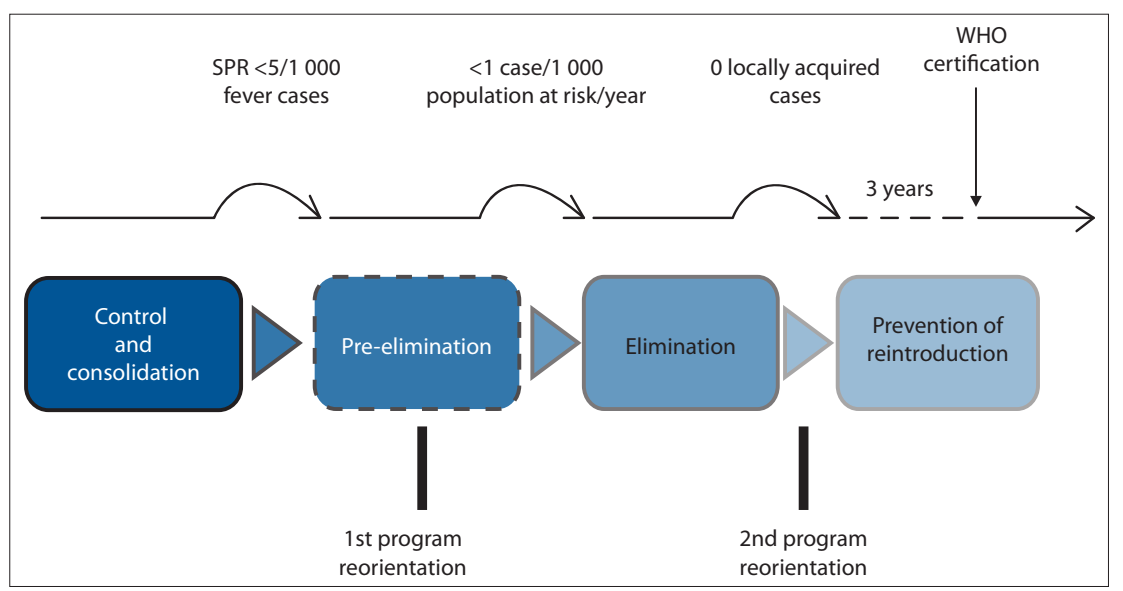

Fig. 2. WHO malaria elimination continuum. ${ }^{[2]}$

administrative structures have resulted in divergent approaches to surveillance, data collection and reporting. The harmonisation of data collection and data management systems and the standardisation of indicators for monitoring and reporting have been identified as necessary prerequisites for achieving elimination. To this end, the newlydeveloped national web-based reporting tool allows for integration of data from the various provincial regional systems to inform core indicators identified as critical to the national elimination campaign. These include malaria positivity rates, confirmed case counts and case fatality rates (CFRs). Integration of the two non-endemic provinces, Gauteng and the North West, into the national reporting systems are currently underway. Furthermore, the national malaria notification form has been redrafted to integrate regional requirements into a single tool. As this updated form is scheduled for rollout in 2013, amendments to the MIS to accommodate these changes are in progress. The NDoH's Malaria Elimination Monitoring and Evaluation Plan 2012 - 2018 and draft Surveillance Guidelines for Malaria Elimination and Prevention of Re-Introduction for South Africa 2012, document the milestones achieved in harmonising provincial tools, strategies and practices over the past three years.

As entomological surveillance is currently conducted irregularly, vector data critical for identifying foci of transmission are not readily available. The lack of capacity and resources at provincial level prevents the collection of reliable data on a regular basis to better inform control activities. Further challenges include capacity development and skills retention to ensure proper case investigation, data management, data analysis and entomological surveillance.

\section{Importation of malaria from malaria- endemic neighbouring countries}

SA shares borders with malaria-endemic Mozambique and Swaziland in the east and Zimbabwe in the north. Imported cases from neighbouring countries and others further north represent $>40 \%$ of the case burden in endemic provinces. ${ }^{[5]}$ Between $40 \%$ and $50 \%$ of the infections reported in KwaZulu-Natal and between $70 \%$ and $80 \%$ of those reported in Mpumalanga are acquired outside SA, with Mozambique accounting for most of the imported cases. In Limpopo, although $<20 \%$ of infections are reported as imported, mainly from Zimbabwe, a further $20 \%$ of reported cases remain unclassified with their origin of infection either inconclusive or the patient proving untraceable during the follow-up investigation. High numbers of imported infections not only contribute adversely to the case burden, but delayed diagnosis in these cases results in higher CFRs in border regions. Identification and treatment of imported cases through border screening remains an elusive target, with high associated costs and the number of informal entries into SA large and often unknown. Feasibility studies scheduled to be conducted during 2013 aim to characterise the precise costs and logistics required to implement an effective border malaria management programme.

\section{Uncontrolled high transmission in neighbouring \\ countries}

Following unprecedented increases in global funding for malaria control and elimination, the burden of malaria in sub-Saharan Africa has decreased dramatically over the past decade ${ }^{[6]}$ However, the reductions in malaria morbidity and mortality have not been uniform in southern African countries, with some countries transitioning to elimination and others experiencing a resurgence of the disease. ${ }^{[7]}$ As a result of marked decreases in malaria transmission, SA is well positioned to move beyond the malaria target of the Millennium Development Goals (MDGs) and progress towards elimination. ${ }^{[8]}$

Fortuitously for SA, all but two of its bordering countries are also targeting elimination. Swaziland is aiming to be the first sub-Saharan mainland African country to eliminate malaria, while both Namibia and 
Botswana have adopted elimination as a policy agenda. ${ }^{[9]}$ Zimbabwe and Mozambique are still in the control phase of the elimination continuum. Malaria cases originating in Mozambique and Zimbabwe have a direct impact on the malaria burden of districts in Limpopo, Mpumalanga and KwaZulu-Natal provinces. ${ }^{[10]}$ Implementing effective cross-border malaria control interventions in these countries would assist these two provinces in transitioning to elimination.

\section{Financial resources}

Achieving zero local malaria cases will require substantial financial resources, some of which will need to be sustained long after elimination is achieved, to prevent the reintroduction of malaria into SA. ${ }^{[2]}$ Since the 1940s, the SA government has largely been responsible for funding for malaria control activities in SA. This has allowed sustained effective control interventions for more than 70 years. Over this period, provincial control programmes have been able to adopt and implement policies that were evidence based and feasible. These programmes have the ability to mobilise financial resources at short notice, as was evident during the 1999/2000 malaria season when R39 million was mobilised to support malaria outbreak containment efforts in Limpopo, Mpumalanga and KwaZulu-Natal. ${ }^{[4]}$
The Malaria Control Programme in SA has prepared a costed malaria elimination plan for the period 2012 - 2018 and the anticipated cost of implementing the elimination strategy is approximately R1.5 billion. The projected shortfall in migrating from a controloriented programme to an elimination programme is estimated to be R620 million. When stratifying malaria elimination costs by intervention areas it is evident that surveillance is the major cost driver, utilising $57 \%$ of the total budget whilst vector control accounts for $21 \%$ of the total costs (Table 2). This funding challenge will need to be bridged to ensure that malaria elimination becomesh a reality. Given the competing funding requirements for other priority programmes in SA such HIV/AIDS, tuberculosis, primary healthcare re-engineering and the National Health Insurance (NHI) system, it will be important for innovative funding solutions to be found to aid the malaria elimination programme. Donor funding will not be the solution, as this has proven unsustainable in many countries. Options should be explored for the malaria programme to integrate its surveillance system into the primary healthcare programmes, currently being supported by the political and executive levels within government. ${ }^{[1]]}$ Improved surveillance will make it possible to adopt more focal approaches to transmission

Table 1. Local malaria incidence by province, district and municipality in South Africa, 2011 - 2012 (NDoH malaria statistics)

\begin{tabular}{|c|c|c|c|c|}
\hline \multirow[b]{2}{*}{ Province } & \multicolumn{4}{|c|}{ District (incident rates, /1 000 population at risk) ${ }^{*}$} \\
\hline & $\begin{array}{l}\text { Control } \\
(\geq 5)\end{array}$ & $\begin{array}{l}\text { Pre-elimination } \\
(<5)\end{array}$ & $\begin{array}{l}\text { Elimination } \\
(<1)\end{array}$ & $\begin{array}{l}\text { Prevention of re-introduction } \\
\text { (0 local cases) }\end{array}$ \\
\hline Limpopo (0.5) & & Vhembe (1.415) & $\begin{array}{l}\text { Mopani }(0.67) \\
\text { Waterberg }(0.042) \\
\text { Capricorn }(0.026) \\
\text { Sekhukhune }(0.005)\end{array}$ & \\
\hline Mpumalanga (0.127) & & & Ehlanzeni (0.294) & $\begin{array}{l}\text { Gert Sibande } \\
\text { Nkangala }\end{array}$ \\
\hline KwaZulu-Natal (0.026) & & & $\begin{array}{l}\text { Umkhanyakude }(0.144) \\
\text { Zululand }(0.044) \\
\text { uThungulu }(0.006)\end{array}$ & $\begin{array}{l}\text { Amajuba } \\
\text { eThekwini } \\
\text { iLembe } \\
\text { Sisonke } \\
\text { Ugu } \\
\text { uMgungundlovu } \\
\text { uMzinyathi } \\
\text { uThukela }\end{array}$ \\
\hline
\end{tabular}

Table 2. Financial gaps for malaria elimination in South Africa, 2012 - 2018

\begin{tabular}{lllllll}
\hline Province & Surveillance & Vector control & $\begin{array}{l}\text { Health } \\
\text { promotion }^{*}\end{array}$ & $\begin{array}{l}\text { Case } \\
\text { management }^{*}\end{array}$ & $\begin{array}{l}\text { Programme } \\
\text { management }^{*}\end{array}$ & Total $^{*}$ \\
\hline Gauteng & 12570280.00 & - & 10809201.00 & 2494275.00 & 3327946.00 & 29201700.00 \\
KwaZulu-Natal & 35969105.00 & 80876914.00 & 19450875.00 & 7327197.00 & 9512701.00 & 153136792.00 \\
Limpopo & 276724215.00 & 36225042.00 & 6689059.00 & 5641483.00 & 2241846.00 & 327521645.00 \\
Mpumalanga & 16545169.00 & 14905111.00 & 32621272.00 & 351016.00 & 3902882.00 & 68325450.00 \\
Northwest & 13309883.00 & 956828.00 & 16821351.00 & 1314044.00 & 329167.00 & 32731273.00 \\
National & - & - & - & - & 8711796.00 & 8711796.00 \\
Total (ZAR) & 355118652.00 & 132963895.00 & 86391757.00 & 17128014.00 & 28026337.00 & 619628656.00 \\
Total (US\$) & 4438983.50 & 16620486.80 & 10798969.60 & 2141001.75 & 3503292.12 & 77453582.00 \\
Financial gap, \% & 57.3 & 21.5 & 13.9 & 2.8 & 4.5 & 100 \\
${ }^{*}$ Prices in ZAR. & & & & & &
\end{tabular}


reduction, thus ensuring that resources are deployed in a more cost-effective manner.

\section{Human resources}

Although many endemic countries have established national malaria control programmes, their effectiveness for scaling up interventions is often constrained because of inadequate human resources at all levels. ${ }^{[12]}$ SA has adopted a preventive and curative approach to malaria control and is thus highly reliant on its human-resource component for implementing the indoor residual spray (IRS) programme and treatment services. The effectiveness of the control programme is often compromised by poorly-trained spray personnel as well as healthcare practitioners who have not been trained properly in diagnosis and treatment aspects of case management. To achieve malaria elimination, it will be necessary to embark on an ambitious capacity-development and training programmes, to overcome these shortages.

\subsection{Training needs}

There is a great need for training of all categories of staff within the malaria control programme, from programme management to persons implementing interventions in the field. As IRS operators are only employed during the malaria season, they are hired on a short-term basis. Unfortunately many trained IRS operators do not return, resulting in refresher training being offered alongside introductory training to ensure both inexperienced and experienced spray operators have a similar level of proficiency. ${ }^{[13]}$

The training of health workers in malaria diagnosis, particularly the use of malaria rapid diagnostic tests (RDTs) is an important component of a wider strategy to improve parasite-based malaria diagnosis at healthcare facilities where microscopy is not readily available. Health personnel benefit from regular training to ensure that they are able to correctly use RDTs and administer artemisinincontaining combination treatments (ACTs). ${ }^{[14,15]}$ A study of Malawian health workers found most participants knew of ACT and malaria treatment guidelines. ${ }^{[16]}$ However, most participants lacked any formal training on both ACT use and adverse ACT effect reporting. These investigators identified a need for more training of healthcare professionals to ensure correct and effective use of ACT. A similar situation currently exists in SA and steps must be put in place to ensure that health workers receive regular refresher training in malaria diagnosis, treatment and reporting.

Information systems developed for use in SA are currently underutilised due to the lack of appropriately qualified staff at a national and provincial level. To rectify this deficiency, recruitment and training is required at all levels of the malaria control programme.

\section{Mitigating identified gaps for progressing the malaria elimination agenda in SA}

For SA to transition towards elimination, the following needs to be in place: effective surveillance systems; early detection and rapid response to epidemics and outbreaks; constant monitoring and evaluation of critical indicators for elimination; and the identification of hotspots and foci of transmission to tackle the parasite and disease more efficiently and effectively.

In addition to addressing the challenges for malaria elimination already identified, it will be imperative to tackle malaria surveillance innovatively for SA to progress more rapidly toward its elimination goals. It will become necessary to detect foci of transmission and hotspots through the use of gametocyte detection or through the use of techniques to determine immune responses. In addition, the use of modelling and forecasting will become useful for determining hotspots, establishing an evidence-based way of targeting IRS to foci of transmission, and containing costs for elimination.

The decline in malaria cases and deaths over the last decade in SA has been well documented, with almost all municipalities in malarious districts reporting incidence levels of $<5$ cases/ 1000 population at risk per annum in $2010 .{ }^{[8]}$ As a result, most areas are either in the elimination or pre-elimination phase.$^{[10]}$ However, transition to malaria elimination needs to be made through a concentration of activities towards identification of residual transmission foci and intensification of efforts to eliminate the last few infections. ${ }^{\left[{ }^{[17]}\right.}$ Low average morbidity rates that are consistent with the elimination or pre-elimination phases do not imply that transmission is uniformly low in these municipalities, since most cases originate in a small number of localities which represent foci of transmission well above the pre-elimination level. Therefore, to make further progress in reducing malaria cases and deaths in the transition to elimination, it will be necessary to adopt more intensive focal approaches, which will be briefly discussed at the end of this article.

Almost the entire recent decline in annual cases and deaths occurred in the first half of the decade to 2010, with no further reductions seen in recent years. The dramatic decline in cases in the years after 2000 was largely attributable to overcoming insecticide and drug resistance that were responsible for the major epidemic in KwaZulu-Natal, and through the control of malaria in Mozambique through the Lubombo Spatial Development Initiative (LSDI). To achieve further reductions in malaria morbidity and mortality will require addressing the gaps in knowledge discussed above. Above all, it will require improved surveillance so that foci of transmission can be rapidly identified and acted upon by deploying prevention and case-finding resources in these hotspots.

During the transition to elimination, there is a heightened risk of malaria epidemics, which has to be mitigated through a programme of epidemic preparedness. Epidemics or outbreaks can occur when malaria attacks vulnerable populations with little or no immunity, such as the populations in SA. In these instances, persons of all age groups are at risk of contracting the disease.

Transitioning towards elimination, therefore, brings about new challenges for the country to be able to scale up surveillance, secure political (financial) commitment and epidemic preparedness and response (EPR), in light of the low number of malaria cases. Within the elimination phase it is essential that all outbreaks be investigated within 2 weeks of detection, highlighting the need for capacity and resources to respond timely and effectively to an outbreak. Continuous monitoring of a district's progress will be critical for the attainment of the elimination goal by 2018 .

As part of the elimination programme, the National Malaria Control Programme needs to work with stakeholders such as the South African Weather Services to establish more accurate predictions of outbreak and epidemic hotspots in malaria-endemic areas, based on entomological and epidemiological surveillance. This information needs to be shared with stakeholders representing all levels of the health system to reduce risk for outbreaks and epidemics.

\section{Monitoring and surveillance}

Key issues in the scaling up of monitoring and surveillance under the elimination campaign include: reporting of passively-detected cases within 24 hours; investigation of these cases within 48 hours; rapid re-active case detection and treatment in communities surrounding passive notifications, identification of foci of local transmission typified by local case episodes, coupled with the presence of suitable vector population; and the mapping of spatial locales of cases, hotspots, transmission foci and outbreaks. Informed, targeted and timeous 
response in terms of case management, community mobilisation and vector control depend critically upon such systems and data.

\subsection{Hotspots}

The identification of hotspots - that is, areas of higher than average malaria transmission - becomes crucial when transitioning from malaria control to malaria elimination. Besides their representing residual sites of malaria transmission, the basic reproductive rate in hotspots can be four times the rate in the larger focus of malaria transmission, ${ }^{[18]}$ posing a severe threat to malaria elimination efforts. Interventions targeting hotspots rather than foci of transmission can potentially reduce community wide malaria transmission, ${ }^{[19]}$ particularly if malaria occasionally spills over from hotspots into the surrounding regions. ${ }^{[20]}$

Identification of hotspots through the measurement of increased exposure to malaria vectors or through annual clinical malaria incidence is inefficient at low transmission intensities. The detection of malaria gametocytes or malaria-specific immune responses in human populations has been shown to be more effective at low transmission intensities. Following the identification of a hotspot, appropriate targeted interventions must be employed as existing interventions are not sufficient to eliminate malaria. ${ }^{[2]]}$ The selected intervention must suit the setting and be cost-effective. ${ }^{[2]}$

As SA proceeds with its elimination agenda, implementation of hotspot mapping and targeted interventions needs to become routine practice. The first step should be serological surveys to determine malaria exposure as well as to identify hotspots in the malaria endemic regions. Based on data generated from the survey, decisions on whether targeted interventions are feasible, cost-effective or necessary, can be made. Possible targeted interventions could include larviciding, active case detection, reactive screening and treatment of individuals living in close proximity to a confirmed malaria case, and focal mass drug administration.

\subsection{Modelling and forecasting}

Mathematical models have been used in malaria research for the purpose of understanding malaria transmission and predicting the impact of specific interventions since the pioneering work of Ronald Ross and later, George MacDonald. ${ }^{[11]}$ During the transition to elimination, malaria modelling will be needed to analyse and predict the effects of new tools and intervention strategies and the costs and cost effectiveness of these strategies. Modelling can be used to gain insights into the effectiveness of combinations of interventions in particular settings before they are implemented. This is important when such combinations are costly, and have not yet undergone trials to evaluate whether they will contribute to the interruption of transmission.

The role of mathematical modelling in the transition to elimination phase has been described by the malERA Consultative Group on Modelling. Such models would be needed to design efficient sampling schemes for identifying foci of transmission in a lowendemicity setting. Models would also be needed to determine the minimum intervention coverage, e.g IRS, that would be required to minimise the risk of malaria epidemics, at the same time as keeping costs at levels that make the transitioning phase sustainable. In SA, models could be used to help fine-tune surveillance and monitoring, by investigating the extent to which active case finding needs to supplement the passive case detection system. This should include modelling the potential transmission reduction that can be achieved with approaches such as focal screening and treatment or highly focal screening and treatment. As in other elimination countries, targeting hotspots of transmission will be an important element of the transition to elimination in SA. Currently, such hotspots are not well defined in malaria areas of SA and models can be used to help identify these hotspots efficiently.

\section{Need for targeting and scaling down of interventions}

Evidence suggests substantial benefits in targeting control activities at foci of transmission, asserting that a break in overall transmission may be more effectively achieved through this approach, especially so where pockets of transmission are evident amid generally decreasing trends in an area. ${ }^{[18]}$ Vector control and surveillance activities were conducted comprehensively across all endemic provinces in SA prior to 2007. With diminishing case numbers in recent years, provincial malaria control programmes have variously phased back activities to concentrate on known areas of high risk. Targeted IRS and active surveillance are now still conducted comprehensively in high-risk districts but to a limited extent in districts where local transmission is suspected to have been interrupted to some extent. Further, in appropriate situations, high costs associated with blanket IRS may be offset by targeting activities and redirecting savings to other critical aspects of the national elimination programme. ${ }^{[23]}$

The identification of foci of clinical and asymptomatic infections and the concentration of activities in hotspots is a key operational strategy during the pre-elimination phase ${ }^{[17]}$ In transitioning from control to elimination, provincial malaria control programmes in SA will need to shift the hub of their activities from generalised essential intervention coverage to a concentration of activities to attack such foci of perpetual transmission. Scaling down generalised control will have to proceed in an evidence-based manner, so as to both minimise the risk of renewed transmission in places where malaria has not been seen for some time, and to minimise the costs. Detecting and treating a very large proportion of any new infections will be necessary if transmission is to be interrupted. This requires highly sensitive passive and active case detection, combined with a rapid response to evidence of local transmission including targeted vector control. ${ }^{[19]}$

The current untargeted approach of IRS in SA's malaria control districts is potentially inefficient in spraying areas where there is little or no evidence of recent transmission. Blanket IRS in this setting may jeopardise programme sustainability, compromise household acceptability and may reduce the quality of spray application. Elimination efforts may be unsustainable if they demand high levels of resources and household compliance in the virtual absence of malaria cases. A more focal approach, limited to localities which have a high probability of recent transmission, would deliver more effective IRS by ensuring closer supervision, more effort in accessing locked premises, and higher acceptability and compliance by householders and communities.

During the malaria elimination and certification phases, substantial additional investments have to be made in highquality, reliable surveillance and response systems, which require the ability to identify record and investigate every single case. This task will be all the more challenging as a result of the large number of imported malaria cases from neighbouring countries, in particular from Mozambique. Such additional investments are difficult to fund if simultaneously rising costs of insecticides for generalised IRS have to be met. Savings resulting from scaling back generalised IRS can be spent on enhanced surveillance. Such enhanced surveillance will still have at its core a reliable passive case detection system, but it will have to be complemented with extensive active surveillance, particularly in conjunction with investigations of passive cases. 


\section{Advocacy and partnerships in moving from malaria control to elimination in SA}

There have been considerable and varied activities with respect to advocacy, health promotion and partnership development in the Malaria Control Programme to date. Although these activities are often not subject to the same scientific scrutiny and rigour as clinical trials to demonstrate their net benefit prior to implementation, they form an important pillar of a successful malaria control programme. ${ }^{[24]}$

Any advocacy, health messaging and partnership strengthening intervention has to be contextualised within: the current milieu of the reduction in malaria literacy in non-endemic areas; the perception of reduced malaria risk among policy makers, healthcare workers and communities; SA's cultural diversity and its migrant populations; community perceptions of IRS and of healthcare in general; the vertical nature of the programme; the re-engineered primary healthcare service delivery model, and importantly, the limited existing resources required to provide a comprehensive, integrated and standardised advocacy and marketing strategy (see Chapter 7). ${ }^{[10}$

\section{Conclusion}

Much has been achieved towards achieving malaria control in SA and setting the country on a path towards elimination. However, the road to elimination will require additional financial and human resources, a paradigm shift in diagnostics and a review of vector control strategies to more targeting approaches, especially within hotspots. Coupled with this is the need to consider the malaria situation in neighbouring countries, given the significant burden of important cases into a receptive environment.

\section{References}

1. Cotter C, Sturrock HJ, Hsiang MS, et al. The changing epidemiology of malaria elimination: New strategies for new challenges. Lancet 2013 (in press). [http://dx.doi.org/10.1016/S0140-6736(13)60310-4]
2. World Health Organization. Malaria Elimination: A Field Manual for Low and Moderate Endemic Countries. Geneva: WHO, 2007. http://whqlibdoc.who.int/publications/2007/9789241596084_eng.pdf (accessed 11 August 2013).

South African National Department of Health. Malaria Elimination Strategy for South Africa 2012 - 2018. Pretoria: $\mathrm{NDoH}, 2012$.

4. South African National Department of Health. National Malaria Programme Review. Pretoria: NDoH, 2009. 5. South African National Department of Health. National Malaria Statistics. Pretoria: NDoH, 2013.

6. World Health Organization. World Malaria Report, 2012. Geneva: WHO, 2012. http://www.who.int/malaria/ publications/world_malaria_report_2012/en/ (accessed 12 August 2013).

7. Mublications/world_malaria_report_2012/en/ (accessed 12 August 2013). Mharakurwa S, Thuma PE, Norris DE, et al. Malaria epidemiology and

8. Moonasar D, Nuthulaganti T, Kruger PS, et al. Malaria control in South Africa 2000 - 2010: Beyond MDG6. 8. Moonasar D, Nuthulaganti T, Kruger PS, et al. Malaria control in Sou
Malar J 2012;11:294. [http://dx.doi.org/10.1186/1475-2875-11-294]

Malar J 2012;11:294. [http://dx.doi.org/10.1186/1475-2875-11-294]
9. Kunene S, Phillips AA, Gosling RD, Kandula D, Novotny JM. A national policy for malaria elimination in Swaziland: A first for sub-Saharan Africa. Malar J 2012;10:313.[http://dx.doi.org/10.1186/14752875-10-313

. Maharaj R, Morris N, Seocharan I, et al. The feasibility of malaria elimination in South Africa. Malar J 2012;11:423. [http://dx.doi.org/10.1186/1475-2875-11-423]

11. MacDonald G. The Epidemiology and Control of Malaria. London: Oxford University Press, 1957:45-62.

12. Soce I, Fall N, Bakyaita N, et al. Accelerated malaria control toward its elimination in the African region. Afr Health Monitor 2010;11:37-43.

13. World Health Organization. Operational Manual on the Application of Insecticides for Control of the Mosquito Vectors of Malaria and Other Diseases. Geneva: WHO, 1997. http://www.who.int/iris/ handle/10665/63702 (accessed 11 August 2013).

14. Moonasar D, Goga AE, Frean I, et al. Primary health workers' proficiency in using malaria rapid diagnostic tests in Limpopo Province. S Afr Med J 2009;99:798-799.

15. Kyabayinze DJ, Asiimwe C, Nakanjako D, et al. Programme level implementation of malaria rapid diagnostic 5. Kyabayinze DJ, Asiimwe C, Nakanjako D, et al. Programme level implementation of malaria rapid diagnostic
tests (RDTs) use: Outcomes and cost of training health workers at lower level health care facilities in Uganda. BMC Public Health 2012;12:291. [http://dx.doi.org/10.1186/1471-2458-12-291]

16. Kalilani-Phiri LV, Lungu D, Coghlan R. Knowledge and malaria treatment practices using artemisinin combination therapy (ACT) in Malawi: Survey of health professionals. Malar J 2011;10:279. [http://dx.doi. org/10.1186/1475-2875-10-279]

7. Moonen B, Cohen JM, Snow RW, et al. Operational strategies to achieve and maintain malaria elimination. Lancet 2011;376:1592-1603. [http://dx.doi.org/10.1016/S0140-6736(10)61269-X]

18. Bousema T, Drakeley C, Gesase S, et al. Identification of hot spots of malaria transmission for targeted malaria control. J Infect Dis 2010;201:1764-1774. [http://dx.doi.org/10.1086/652456]

19. Bousema T, Griffin JT, Sauerwein RW, et al. Hitting hotspots: Spatial targeting of malaria from control and elimination. PLoS Medicine 2012;9: e1001165. [http://dx.doi.org/10.1371/journal. pmed.1001165]

20. Bousema T, Stevenson J, Baidjoe A, et al. The impact of hotspot-targeted interventions on malaria transmission: Study protocol for a cluster-randomized controlled trial. Trials 2013;14:36. [http://dx.doi. org/10.1186/1745-6215-14-36]

21. Griffin JT, Hollingsworth TD, Okell LC, et al. Reducing Plasmodium falciparum malaria transmission in Africa: A model-based evaluation of intervention strategies. PLoS Med 2010;7:e1000324. [http:// dx.doi.org/10.1371/journal.pmed.1000324]

. Woolhouse ME, Dye C, Etard JF, et al. Heterogeneities in the transmission of infectious agents: Implications for the design of control programs. Proc Natl Acad Sci 1997;94:338-342. [http://dx.doi.org/10.1073/pnas.94.1.338 ] 23. Teklehaimanot A, McCord GC, Sachs JD. Scaling up malaria control in Africa: An economic and epidemiological assessment. Am J Trop Med Hyg 2007;77:138-144.

24. Gillon R. Health education and health promotion. J Med Ethics 1987;13:3-4. [http://dx.doi. org/10.1136/jme.13.1.3] 